Revue des patrimoines

\title{
Actualité du recensement des peintures murales en Bourgogne : PACoB
}

Marie-Gabrielle Caffin, Françoise Perrot et Alain Rauwel

\section{OpenEdition}

Journals

Édition électronique

URL : http://journals.openedition.org/insitu/10805

DOI : $10.4000 /$ insitu. 10805

ISSN : 1630-7305

Éditeur

Ministère de la culture

Référence électronique

Marie-Gabrielle Caffin, Françoise Perrot et Alain Rauwel, « Actualité du recensement des peintures murales en Bourgogne: PACoB », In Situ [En ligne], 22 | 2013, mis en ligne le 13 novembre 2013, consulté le 19 avril 2019. URL : http://journals.openedition.org/insitu/10805; DOI : 10.4000/ insitu. 10805

Ce document a été généré automatiquement le 19 avril 2019.

\section{(1) $\Theta$}

In Situ Revues des patrimoines est mis à disposition selon les termes de la licence Creative Commons Attribution - Pas d'Utilisation Commerciale - Pas de Modification 4.0 International. 


\title{
Actualité du recensement des peintures murales en Bourgogne: PACoB
}

\author{
Marie-Gabrielle Caffin, Françoise Perrot et Alain Rauwel
}

1 La peinture murale est, depuis plusieurs années, l'objet d'une attention toute particulière dans la région Bourgogne ${ }^{1}$. Elle s'est manifestée à travers les nombreux chantiers de conservation-restauration mis en place depuis la fin des années 1980 et les différentes campagnes de recensement entreprises, également, à cette période. L'actualité continue à nourrir notre connaissance de ce patrimoine puisque de nouvelles découvertes et/ou restaurations sont signalées très régulièrement. Les deux enquêtes qui se sont déroulées essentiellement sur le terrain en 1988-1992 et 2000-2002, ont permis de recenser environ 800 édifices sur les quatre départements (Côte-d'Or, Nièvre, Saône-et-Loire et Yonne) qui constituent la région Bourgogne. Depuis 2004, l'association Patrimoine, Ambiances et Couleurs de Bourgogne, $\mathrm{PACOB}$, a souhaité tenir à jour cet inventaire tout en l'accompagnant d'un important travail d'information sur le terrain, de valorisation et de publications.

\section{Genèse du projet}

2 En 1986, le vitrail avait fait l'objet d'une première campagne d'inventaire thématique menée en Bourgogne. Celle-ci s'inscrivait dans un projet national, le Recensement des vitraux anciens de la France, placé sous l'égide du Corpus vitrearum. Le Conseil régional, fort de cette première expérience positive, décide de mettre en place l'inventaire thématique de la peinture murale. C'est en 1988 que débute la première opération de recensement menée par le service régional de l'Inventaire de Bourgogne (DRAC Bourgogne), maître d'œuvre. Le service des Monuments historiques (DRAC Bourgogne) a également collaboré scientifiquement à ce projet, la conservation-restauration des peintures murales étant au cœur de ses préoccupations à cette époque.

Dans les années 1952-1953, A. Neury (C.R.M.H.) ${ }^{2}$ avait déjà parcouru la Bourgogne en référençant les sites des peintures murales dans un premier véritable corpus accompagné 
d'une campagne photographique. Dans les publications nationales de P. Deschamps et M. Thibout pour la période romane et gothique ou encore d'Y. Bonnefoy ${ }^{3}$ pour ne citer que ces références majeures, la peinture murale bourguignonne occupait, dans ces mêmes années, une place non négligeable. Cependant, depuis lors, les mentions de peintures murales apparaissaient localement de façon sporadique, essentiellement dans les revues savantes au travers de notices ou courtes monographies publiées au gré des découvertes. Une enquête régionale avait donc toute sa raison d'être pour acquérir une meilleure connaissance de ce patrimoine mural.

Lors de la première campagne de recensement en 1988-1992, le Conseil régional a adressé, en amont, à toutes les communes de Bourgogne, un questionnaire visant à recenser les peintures murales présentes dans les édifices civils et religieux sur l'ensemble du territoire. Dans un même temps, l'inventaire d'A. Neury ainsi qu'une partie des publications locales (bulletins, mémoires, journaux...) et les dossiers préexistants du Service régional de l'Inventaire issus des enquêtes topographiques, ont été dépouillés, toujours dans le but de dresser une première liste de sites à visiter. Une chargée d'étude a été recrutée et formée pour travailler sur le terrain avec un photographe professionnel du service. Une fiche technique a été élaborée spécialement pour cette enquête, servant à établir les dossiers papier en vue de la mise en ligne d'une partie des fiches sur la base Palissy, base des objets mobiliers. Cette fiche avait été conçue selon trois grandes parties. La première était consacrée à l'identification de la peinture murale (titre, localisation dans l'édifice, dimensions...) et du lieu de conservation (nom de la commune, du département, de l'édifice et de sa date), la deuxième partie avait pour but de rassembler des données techniques sur la peinture murale (stratigraphie, technique et couleurs employées par le peintre, mention des rapports d'intervention et constat d'état) et la dernière grande partie était réservée à l'étude de la peinture (iconographie, style, relevé des inscriptions et datation). Une bibliographie complétait cette fiche. Pour chaque décor, scène ou groupe de scènes, une fiche était établie. Chaque site visité et ses peintures ont fait l'objet d'une campagne photographique très détaillée (plus de 9000 clichés).

5 Cette enquête thématique, réalisée en 20 mois, a permis de recenser environ 500 édifices possédant des décors muraux s'étendant du haut Moyen Âge au XXe siècle. Le Conseil régional souhaitait clore cette opération par la réalisation d'une exposition et d'un catalogue, le Musée archéologique de Dijon en a été le lieu d'accueil et le conservateur, le commissaire de l'exposition, en lien avec le Service régional de l'Inventaire. Dans le catalogue associé, D'Ocre et d'azur. Peintures murales en Bourgogne $e^{4}$, les premières synthèses ont pu être rédigées, accompagnées de plus d'une soixantaine de notices et de la liste exhaustive des édifices à peintures recensés dans un très bref descriptif.

6 Le corpus a été en grande partie versé dans la Base Palissy, base nationale du ministère de la Culture <lien vers http://www.culture.gouv.fr/culture/inventai/patrimoines. Consultable sur internet, il permet d'obtenir un résumé des fiches papier; en revanche les clichés numérisés ne sont pas encore versés à ce jour.

7 En 2000-2001, l'association PACoB (créée en 1998) décide de mettre dans ses priorités la reprise de l'enquête pour faire le point sur les nouvelles découvertes et restaurations. Elle reprend pour cela contact avec le Service régional de l'Inventaire de Bourgogne. Deux étudiantes de l'Université de Bourgogne (U.M.R. 5594) sont chargées d'établir une première liste des découvertes récentes. Il apparaît nécessaire, au vu de leur rapport de stage, de mettre à jour la première enquête. Une convention est établie entre le Service régional de l'Inventaire de Bourgogne, l'Université de Bourgogne, le CNRS via le Centre 
d'Études médiévales d'Auxerre, et l'association PACoB. Le Conseil régional, particulièrement intéressé par la reprise et le complément de cette enquête, signe une convention directement avec l'association. Le Service de l'Inventaire de Bourgogne met à nouveau à disposition sa compétence scientifique d'enquête et d'étude, son matériel, ses locaux et un photographe professionnel. L'Université de Bourgogne prend en charge l'exploitation de ce travail dans le cadre de sa mission d'enseignement et de liens entre chercheurs. Une chargée d'étude est engagée pendant quelques mois pour cette opération par $\mathrm{PACoB}$ qui œuvre déjà pour la promotion des recherches auprès d'un large public. L'association assurera la gestion financière de l'ensemble de cette opération, apportera des conseils scientifiques sur le terrain et lors du montage de l'exposition itinérante. Elle sera partie prenante dans la réalisation du catalogue dont elle assurera l'édition et sera moteur dans les échanges interprofessionnels.

8 Cette nouvelle enquête a permis d'intégrer les édifices "oubliés» (signalés lors de la présentation de la première exposition), les nouvelles découvertes et les restaurations achevées depuis le précédent recensement ou en cours. Ce sont près de 360 édifices qui seront ainsi ajoutés à la première liste et devront être intégrés à la base du ministère de la Culture. La période couverte a été identique à la première avec l'intégration de nouvelles créations comme les peintures murales réalisées par Dominique Kaeppelin à la chapelle Guillaume de Volpiano, bénite en 1994 à la cathédrale Saint-Bénigne de Dijon. Cet artiste du Puy, spécialisé dans l'art sacré, a conçu dans sa globalité l'aménagement de cet espace et de son mobilier liturgique, à savoir l'ambon, l'autel, la réserve eucharistique, le siège du célébrant, jusqu'au décor mural (fig. $\left.\mathbf{n}^{\circ} \mathbf{1}\right)$. Cet ensemble est empreint de sobriété, d'élégance dans une unité parfaite où tout le discours se concentre autour du mystère de la mort et de la résurrection célébré sur l'autel, et de ceux qui ont éclairé la foi. Il joue sur la simplicité des formes, des images, des figures, les tonalités de blancs, gris pâles, teintes légèrement rosées, le tout rehaussé par l'emploi de l'or, réservé aux auréoles et au mobilier sacré, plus discret pour le siège du célébrant. Au-dessus de l'autel, la Trinité évoque l'icône célèbre d'Andrei Roublev du $\mathrm{XV}^{\mathrm{e}}$ siècle. La figure de l'apôtre saint Jean conduit à suivre son regard, visage dirigé vers le haut, et sa main droite levée pour contempler cette Trinité rendue eucharistique par la présence du pain et du vin. Sur le mur opposé, la Transfiguration fait référence, nous dit Alain Rauwel, historien de l'Université de Bourgogne, «à l'épisode cher aux grands abbés bénédictins de l'âge d'or de Saint-Bénigne, les XI ${ }^{\mathrm{e}}$-XII ${ }^{\mathrm{e}}$ siècles ». 


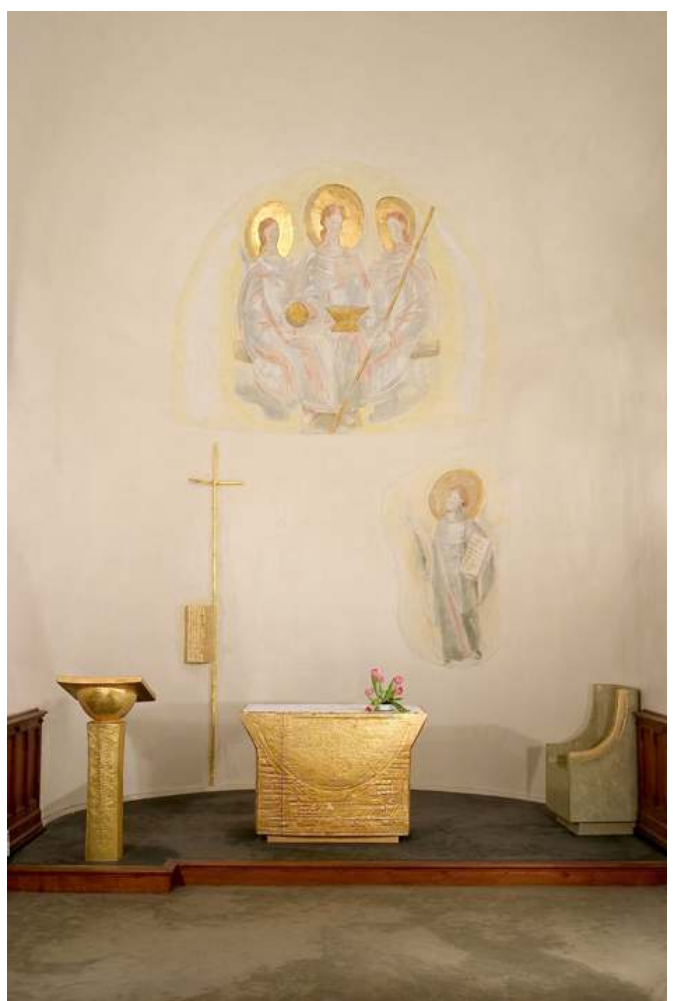

Dijon (21), cathédrale Saint-Bénigne, chapelle Guillaume de Volpiano.

Phot. Roze, Jean-Pierre. (c) Roze, Jean-Pierre.

Ce fut également l'occasion de découvrir ce que laissaient présager les sondages de 1990 dans la chapelle fondée par Jean Caron en 1514 dans l'église paroissiale Notre-Dame de Cuisery, en Saône-et-Loire, à savoir une chapelle entièrement peinte au début du XVI ${ }^{\mathrm{e}}$ siècle où les figures des prophètes dominent au côté de la Résurrection de Lazare (fig. $\mathbf{n}^{\circ} \mathbf{2}$ ). Les prophètes sont regroupés par deux sur le chambranle de la porte au sud et sur les trois autres parois. On peut identifier, entre autres, David et Isaïe. Placés au-dessus de l'autel, à l'est, sous un dais rouge ouvert, ils encadrent un retable aujourd'hui disparu. Sur le chapiteau du dais, l'inscription «Eli, eli, lama sabachthani? $»^{5}$ renvoie aux paroles du Christ sur la croix. Sur le mur sud, la Résurrection de Lazare occupe tout l'espace. La scène exécutée en 1530, selon l'inscription qui l'accompagne, présente l'instant où Jésus fait ouvrir le tombeau de son ami et lui ordonne de sortir. Les marques attestant la présence d'une console placée au niveau du tombeau ouvert permettent de penser qu'une statue représentant Lazare devait compléter ce panneau, mettant en évidence le lien étroit entre la peinture murale et la sculpture. 
Figure 2

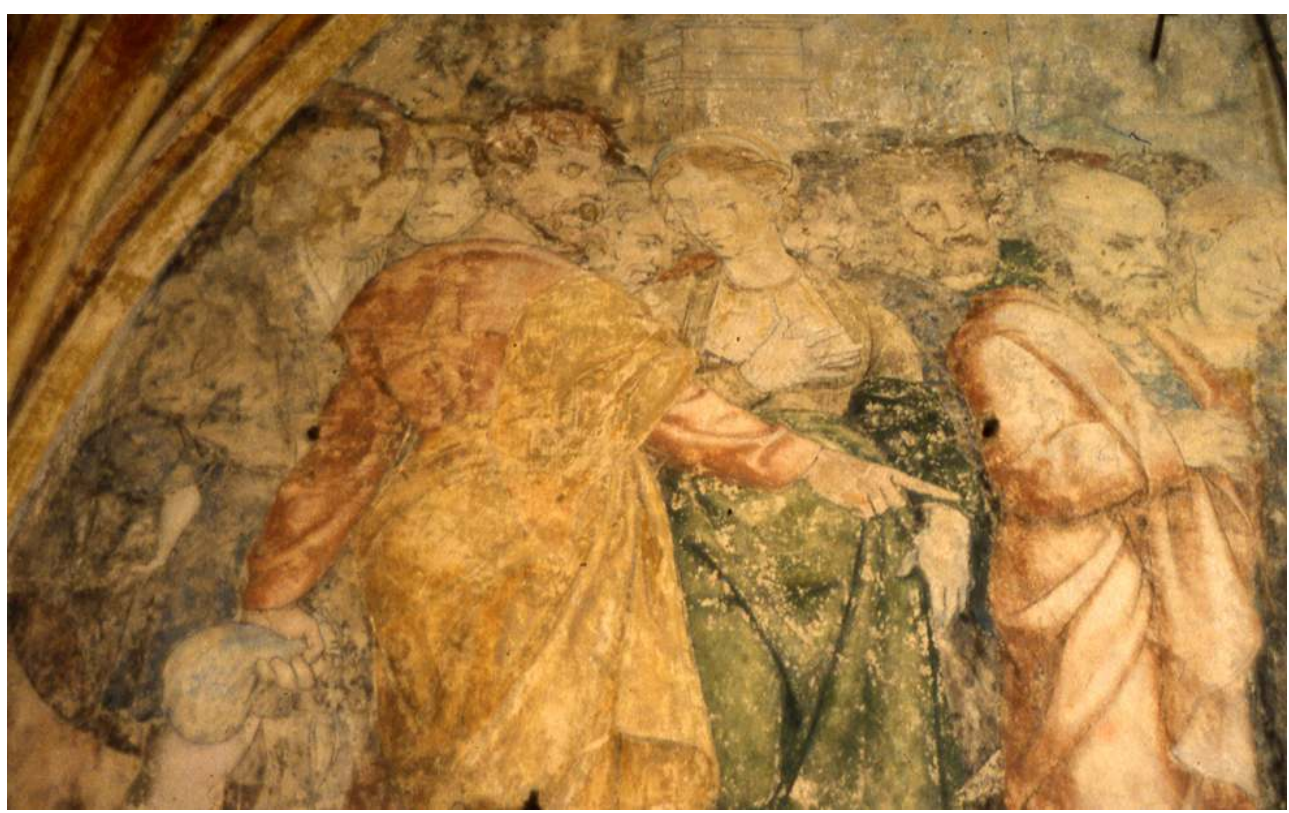

Cuisery (71), chapelle Caron, la Résurrection de Lazare.

Phot. Caffin, Marie-Gabrielle. (c) Caffin, Marie-Gabrielle.

Le bilan de ce complément d'inventaire a été présenté à l'occasion d'une exposition organisée par le Musée archéologique de Dijon et complétée par un catalogue portant un titre unique, Couleur de temps, fragments d'histoires. Peintures murales en Bourgogne $\mathrm{XII}^{e}-\mathrm{XX}{ }^{e}$ siècles ${ }^{6}$. Un colloque a été organisé conjointement à cette première manifestation à l'Université de Bourgogne sous le titre Peintures murales médiévales, XII ${ }^{e}$-XVIe siècle. Regards comparés ${ }^{7}$. L'association PACoB a monté en parallèle une exposition itinérante s'appuyant sur les textes et photographies de la première. Le but recherché était d'aller au plus près du public en la faisant circuler dans toute la Bourgogne. Elle est, encore aujourd'hui, louée aux institutions (musées, bibliothèques...) et prêtée aux associations. C'est un succès à plusieurs titres puisque depuis 2004 et sans discontinuer, un très large public a pu se familiariser avec ce patrimoine, découvrir ses richesses, sa fragilité, comprendre les interventions des professionnels pour sa conservation, sa mise en valeur et enrichir le corpus grâce à la mention de nouvelles découvertes.

\section{Le recensement actuel : PACoB}

Depuis dix ans, l'association a été attentive à développer un partenariat, au service de la valorisation de ces décors muraux. Ce travail bénévole s'effectue, sur le terrain, en lien avec les collectivités territoriales, les services de la DRAC, les associations locales et les professionnels dans le but de conseiller, d'étudier et de présenter ce patrimoine mural. Il est, également, l'occasion pour chacun des membres issus du milieu des chercheurs en histoire de l'art, spécialistes des peintures murales, du vitrail, des conservateurs de musée, des archéologues antiquisants et médiévistes, d'historiens universitaires, de conservateurs-restaurateurs en peintures murales, d'amateurs éclairés et passionnés, d'échanger sur la place de ces décors peints dans notre patrimoine régional, voire national. 
Il n'est pas question d'attendre à nouveau dix ans pour compléter l'inventaire et réunir les spécialistes. L'association se donne pour mission, depuis 2003, de tenir à jour, à la mesure de ses moyens, le fichier des nouvelles découvertes et des restaurations. Le réseau tissé peu à peu par chacun des membres et la présentation de l'exposition itinérante amènent toujours de nouveaux contacts et permettent cette mise à jour régulière. Cet inventaire se présente actuellement sous forme de notices. L'auteur, en charge de la rédaction, effectue un travail sur le terrain, réunit la documentation, relative aux peintures et à leur support, et réalise une couverture photographique. La publication des recherches régionales et le complément de l'inventaire se font par l'intermédiaire des Cahiers $P A C O B^{8}$ en fonction de la disponibilité des auteurs et des financements. Le travail d'inventaire ainsi que la publication sont aujourd'hui autonomes du point de vue financier, grâce à l'engagement des membres de l'association et aux généreux mécènes. Trois Cahiers ont vu le jour depuis 2006 avec, dans le premier, le complément du recensement pour la Nièvre, dans le deuxième celui de l'Yonne et pour le troisième, paru en décembre 2010, celui de la Côte-d'Or. La Saône-et-Loire fait l'objet actuellement d'une réactualisation qui devrait paraître prochainement. Lors de la mise à jour de l'inventaire pour la Côte-d'Or en 2009, où une vingtaine de sites ont fait l'objet d'une notice, nous avons pu constater combien il est nécessaire d'être toujours présent au cœur de l'actualité pour pouvoir faire partager les regrets d'une disparition et les bonheurs d'une apparition.

À l'église Saint-Symphorien de Nuits-Saint-Georges, datée du début du XIII ${ }^{e}$ siècle avec différents remaniements au cours des siècles suivants, les peintures murales sont connues pour partie depuis 1892 et classées M.H. le 5 décembre 1908. En 1953, A. Neury signale la présence de plusieurs panneaux peints et $C$. Hurault est mandaté pour réaliser une campagne photographique. Il réalise alors un cliché de la Crucifixion présente sur un pilier du bras nord du transept et trois clichés de la scène du Martyre de sainte Christine ${ }^{9}$. En 1954, des relevés à l'aquarelle de la scène de martyre sont commandés par le Centre de recherches des Monuments historiques à A. Regnault.

Lors de l'inventaire de 1988-1992, ces deux scènes sont à nouveaux mentionnées et photographiées par le Service régional de l'Inventaire de Bourgogne. S'y ajoutent de nouvelles prises de vue : le Christ au Jardin des oliviers, le Baptême du Christ, les croix de consécration et enfin un donateur avec son saint patron. Un constat d'état est effectué à cette occasion pour l'ensemble des panneaux.

De 2006 à 2007, Éric Pallot, architecte en chef des Monuments historiques et maître d'œuvre, est en charge de la restauration de l'édifice. À cette occasion, les entreprises Eschlimann et Arcams, mandatées pour nettoyer les panneaux visibles, effectuent également des sondages dans le chœur, à l'occasion de la dépose du maître-autel, pour restauration. Ils découvrent trois campagnes picturales différentes, dégagent et consolident les peintures des XIII ${ }^{\mathrm{e}}$-XIV ${ }^{\mathrm{e}}$ siècles et celles de la fin $\mathrm{du} \mathrm{XV}^{\mathrm{e}}$ siècle qui seront ensuite masquées lors de la repose du maître-autel. En revanche, ils termineront par la réintégration des peintures du XVII ${ }^{\mathrm{e}}$ siècle qui resteront visibles. Il est regrettable que les panneaux cachés aujourd'hui n'aient pas fait l'objet d'une étude attentive lors du chantier de restauration. Ces peintures, à peine visibles, représentent, sous l'oculus du chevet, l'Adoration des mages et la Fuite en Égypte (fig. $\mathbf{n}^{\circ} \mathbf{3}$ ) de la fin du XIII ${ }^{\mathrm{e}}$ siècle ou début du XIV siècle à en juger par les clichés. Le lien entre ces scènes et le décor ornemental se développant sur les arêtes des voûtes et sur le mur nord, dans ce même espace, ne peut plus être établi. 
Figure 3

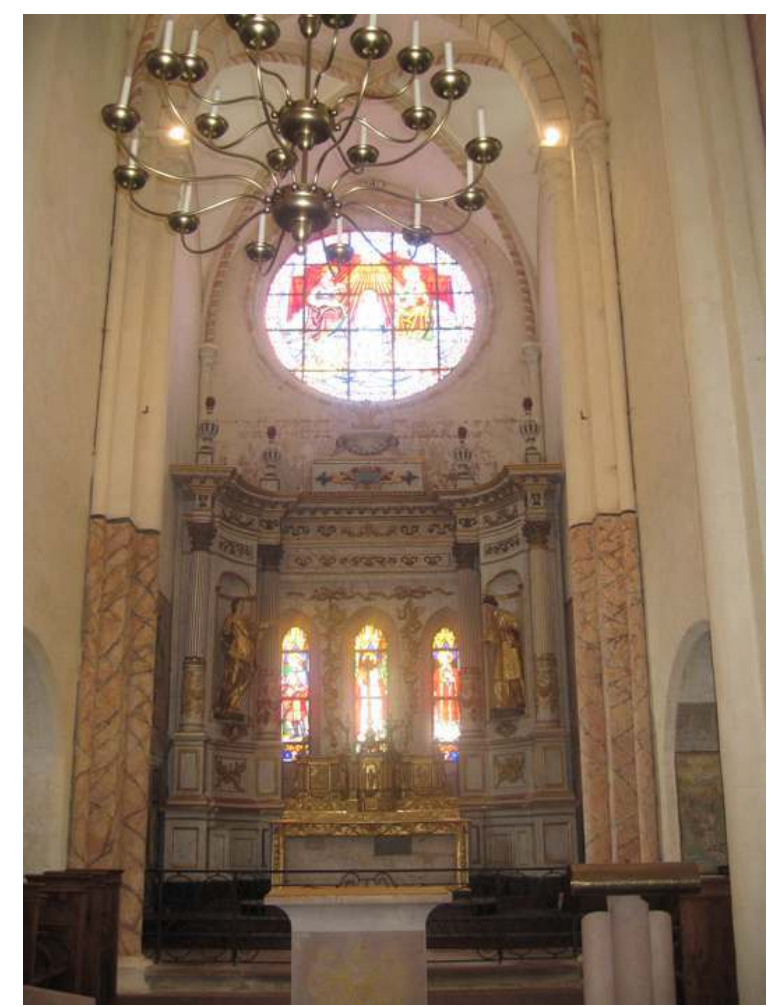

Nuits-Saint-Georges (21), vue générale du chœur.

Phot. Caffin, Marie-Gabrielle. (c) Caffin, Marie-Gabrielle.

Les scènes de la fin du $\mathrm{XV}^{\mathrm{e}}$ siècle, cachées dans leur totalité, étaient consacrées à la Visitation (identification mise en doute et ne pouvant plus être démontrée sur place aujourd'hui) et à la représentation, unique en peinture murale et en Bourgogne, de la Vision de saint Vorles, saint bourguignon. Lors de la pose des nouveaux enduits intérieurs, il a été décidé de faire disparaître le Baptême du Christ présent dans la nef, en très mauvais état et à peine visible. Le cliché pris en 1989 reste aujourd'hui le seul témoin de sa présence et démontre, s'il en est besoin, l'importance de ce travail d'inventaire sans cesse à renouveler. De même, il est regrettable que l'on n'ait pas procédé à une campagne photographique « officielle » et à l'étude par un historien de l'art, avant la repose du maitre-autel du XVIII ${ }^{e}$ siècle, cachant tout ou partie des peintures du XIII ${ }^{e}$-XIV ${ }^{e}$ siècle et celles de la fin du XV siècle. Nous n'avons pu constater que le résultat à la fin du chantier quand nous nous sommes rendus sur les lieux à la demande de Marie-Antoinette Stroeker ${ }^{10}$ de la Commission d'Art Sacré de la Côte-d'Or dans le cadre des échanges de compétences que nous entretenons.

Dans l'Yonne, les peintures murales exécutées par le peintre Zanot en 1782 dans l'église Notre-Dame de l'Assomption de Villeneuve-les-Genêts apparaissaient sous un fond bleu posé au XIX siècle et avaient fait l'objet d'une notice en 1992 dans le catalogue D'ocre et d'azur'11. En 2006, l'atelier ARCOA est chargé de réaliser des sondages sur l'ensemble des murs de l'édifice pendant les travaux de réfection intérieure de l'édifice. Ce fut l'occasion d'établir une stratigraphie précise des enduits peints. Ainsi, sous la signature du peintre Zanot, apparait un cartouche mentionnant la date de 1673 puis, dessous, une autre couche plus ancienne où l'on peut identifier, au centre du chevet, un cheval levant la 
patte antérieure droite probablement exécuté peu après la reconstruction de l'édifice au milieu du XIV ${ }^{\mathrm{e}}$ siècle. Cécile Ullmann, conservateur des Monuments historiques à la Direction régionale des Affaires culturelles de Bourgogne, en charge de l'édifice, avait évoqué dans le $n^{0} 2$ des Cahiers $P A C O B^{12}$, la restauration des peintures murales de cette église, soulignant le lien fort entre la peinture et son support, voire se substituant à celleci dans le cadre de Villeneuve-les-Genêts, "pour le faire disparaître sous un vaste trompe-l'œil ». Cette collaboration avec Cécile Ullmann, en charge du patrimoine mobilier de 2006 à 2011, s'est traduite, outre sa participation à la publication, par une demande auprès des recenseurs de son service de nous tenir informés des nouvelles découvertes ou restaurations et par quelques visites conjointes sur le terrain pour pouvoir confronter nos avis face à des choix de restaurations dans des édifices classés ou inscrits, notamment à l'église de Branches (89) ou encore à celle de Chassignelles (89).

Saint-Jean-Baptiste de Chassignelles, église des XII ${ }^{e}$-XIII ${ }^{e}$ siècles (Cl. M.H. 29 avril 1971) a fait l'objet en 1980 de sondages par Angelescu, conservateur-restaurateur. Une scène de l' Annonciation est découverte et mentionnée dans le catalogue Couleur de temps, fragments d'histoires ${ }^{13}$. En août 2004, Emmanuelle Paris, conservatrice-restauratrice, débute une campagne de dégagement des badigeons modernes de l'intérieur de l'édifice, qui fait apparaitre des décors ornementaux couvrant essentiellement les voûtes tandis que des médaillons contenant des apôtres au Credo et une litre funéraire se développent sur toute la surface des murs de la nef et du chœur (fig. $\left.\mathbf{n}^{\circ} \mathbf{4}\right)$. Ces décors correspondent à plusieurs campagnes d'ornementation de l'édifice. La couche picturale la plus ancienne appartient vraisemblablement aux XIII ${ }^{\mathrm{e}}$-XIV ${ }^{\mathrm{e}}$ siècles. Un Christ est à peine visible sur le mur du chevet. Il est accompagné, sur les parties médianes des murs, des apôtres formant le cortège apostolique. Inscrits sous des arcatures, ils tiennent le livre dans la main gauche et marchent pieds nus, sur une large bordure ornée de deux rubans plissés entrecroisés. Un motif d'appareil imbriqué jaune surligné de rouge semble venir s'accrocher à la bordure pour garnir l'espace inférieur du mur et s'étendre au-delà du chœur, sur les murs de la nef où il est encore perceptible à certains endroits et recréé à d'autres. Il participe à l'ornementation générale de l'édifice, en corrélation avec le décor ornemental des voûtes qui s'organise de manière à individualiser chaque travée tout en s'enrichissant en progressant vers l'est pour se terminer par une suite de médaillons encastrés dans un système de caissons en trompe-l'œil et garnis de lions et d'aigles. 
Figure 4

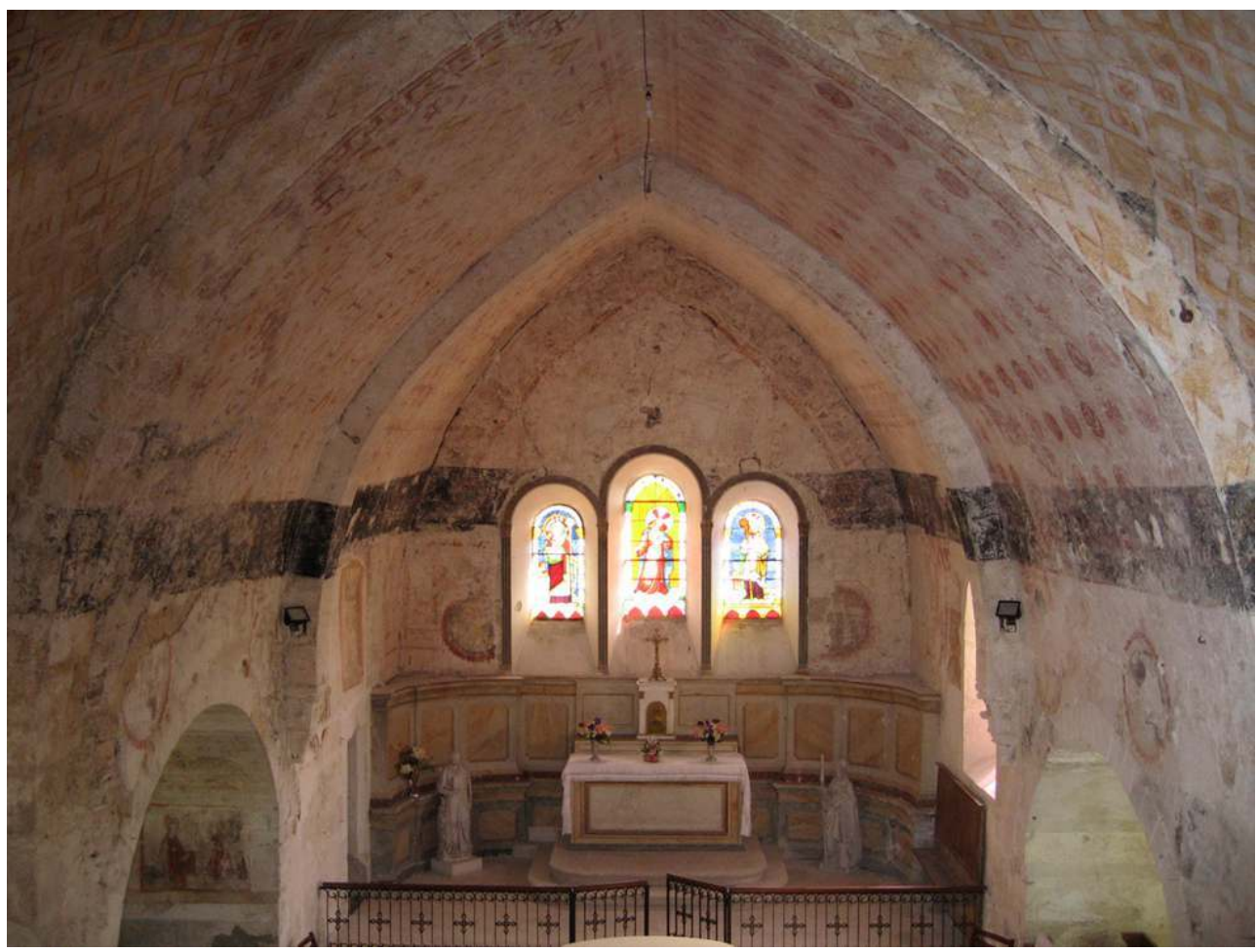

Chassignelles (89), vue d'ensemble de l'intérieur de l'église.

Phot. Caffin, Marie-Gabrielle. (c) Caffin, Marie-Gabrielle.

$20 \mathrm{Au} \mathrm{XVI}$ siècle, douze médaillons sont venus se positionner sur le décor précédent. Ils contiennent les bustes des apôtres du Credo tenant chacun leur attribut. Seuls les apôtres Thomas avec son équerre, Philippe avec sa croix, André avec la croix en X, sont identifiables. Placé dans le chœur de l'église, saint Jean l'évangéliste est également enchâssé dans un médaillon coupé dans sa partie supérieure par celui d'un apôtre non identifiable lui-même étant perturbé par une autre couche picturale. Une litre funéraire se développe sur les murs intérieurs de l'église. Elle a été datée par la présence des armoiries du marquis François Michel Le Tellier de Louvois «d'azur à trois lézards d'argent en pal, au chef de gueules chargés de 3 étoiles d'or ", mort en 1691. Comment mettre en valeur cet ensemble exceptionnel, unifier les enduits restaurés pour une meilleure lisibilité, garder ou recouvrir certaines scènes très ténues, tels ont été les sujets débattus avec la conservatrice des Monuments historiques. L'association des Amis de l'église Saint-Jean-Baptiste et de la sauvegarde du patrimoine de Chassignelles a été, à partir de sa création en 1999, un élément moteur pour la restauration des peintures murales. Des liens précieux se sont tissés entre ses membres et PACoB et des échanges présentation dans ce lieu de l'exposition itinérante, conférence, visites formelles ou informelles - ont permis aux uns et aux autres de mieux connaître ce patrimoine pictural bourguignon. Un article est en cours de rédaction pour le prochain numéro des Cahiers $P A C o B$. Les associations sont donc des relais indispensables entre les institutions et le travail sur le terrain. Elles ont besoin d'être reconnues et soutenues dans leurs démarches patrimoniales. La collaboration avec les différents acteurs de la mise en valeur du patrimoine permet d'actualiser le travail de recherche sur et autour de la peinture murale. Notre crédibilité auprès des collectivités territoriales a peu à peu grandi au fil des 
ans et des échanges engagés. Il peut arriver quelquefois que les Conseils généraux ou le Conseil régional requièrent les services de l'association dans le but de les informer sur un chantier à venir ou de leur apporter de l'aide dans les choix à effectuer de tel ou tel site dans le cadre d'une publication ${ }^{14}$.

Les communes rurales sont demandeuses d'informations, de conseils et d'aide, face à des découvertes souvent inattendues, lors de travaux de réfection intérieure d'une église ou d'une chapelle. Les problèmes liés à la découverte de peintures murales en milieu rural sont multiples et d'autant plus difficiles à gérer que la population est peu nombreuse et les ressources en conséquence. Il en est de même pour les propriétaires privés. Nous essayons d'être présents sur le terrain afin de répondre aux demandes de conseils sur les procédures à suivre pour la mise en valeur des décors peints, sur les aides financières possibles, demandes de subventions publiques ou associatives ${ }^{15}$, et enfin nous apportons les connaissances techniques et stylistiques.

À Diennes-Aubigny, petite commune rurale du sud de la Nièvre, la mairie et l'association Pérennité de l'église de Diennes-Aubigny, créée lors de la découverte des peintures murales, ont fait appel à la fédération SMBS-REMPART Bourgogne ${ }^{16}$ pour monter six chantiers bénévoles sous la responsabilité de Laurence Blondaux, conservatricerestauratrice de peintures murales. Dans l'église Saint-Pierre-Saint-Paul, du XII siècle avec ajout de deux chapelles latérales au XVe siècle, inscrite M.H. le 23 décembre 1964, le décor ornemental composé de claveaux peints a pris possession des encadrements de fenêtres, des arcs et piliers. Des fleurs à cinq lobes typiques de la seconde moitié du XIII ${ }^{\mathrm{e}}$ et du XIV siècle, encore visibles à quelques endroits, parsèment les murs, accompagnées d'étoiles à six branches noires et de joints peints en rouge sur fond crème. Une figure monumentale de saint Christophe a été dégagée sur le mur nord de la nef. Notre rôle a été, à la demande de la mairie et de l'association, de suivre le chantier sur place et d'essayer de dater les peintures, de les intégrer dans un contexte de création bourguignon et d'enrichir la très courte notice parue à l'occasion des sondages de 2002 dans le catalogue Couleurs de temps, fragments d'histoire.

Le maire de la commune d'Asnois (58) a signalé à l'association la découverte, à l'occasion de sondages effectués par Jean-Rémy Brigand, restaurateur, de peintures murales dans l'église Saint-Loup. Ces sondages s'inscrivaient dans un programme de remise en état de l'édifice qui s'ouvrait en deux. À ces travaux architecturaux de grande ampleur pour cette petite commune rurale, est venue s'ajouter la mise au jour d'un premier décor représentant un saint Christophe monumental placé sur la gauche de l'arc triomphal, face à l'entrée principale. Peu à peu, des décors du milieu XIII ${ }^{\mathrm{e}}$ ou début XIV ${ }^{\mathrm{e}}$ siècle, puis un ange de la fin du $\mathrm{XV}^{\mathrm{e}}$ - début du XVI $\mathrm{X}^{\mathrm{e}}$ siècle, apparus dans le bas-côté sud, ont pu être dégagés et consolidés en attendant patiemment la dernière étape de restauration, la réintégration. Dans le chœur (fig. $\mathbf{n}^{\circ} 5$ ), deux scènes ont été conservées suite à leur dégagement malgré leur état d'usure avancé, ne permettant pas leur identification. L'intérêt de ces «traces » iconographiques a pu être précisé lors d'échanges et ainsi aboutir à ce choix de conservation louable qui s'est parfaitement intégré dans la mise en valeur de cet espace. 
Figure 5

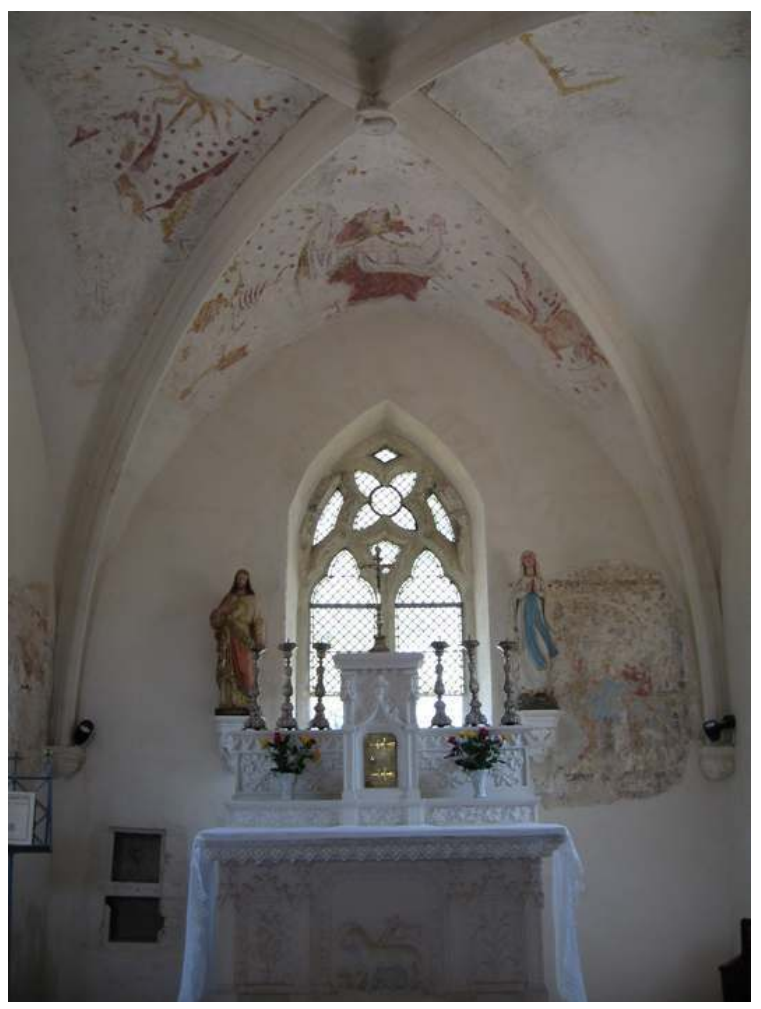

Asnois (58), vue générale du chœur après l'achèvement de la restauration.

Phot. Caffin, Marie-Gabrielle. (c) Caffin, Marie-Gabrielle. réalisé : à l'est, le Christ du Jugement dernier, montrant ses plaies, est assis sur un arc-enciel, accompagné des quatre symboles des évangélistes ; à l'ouest, saint Michel terrasse de sa lance le dragon qui lui agrippe la jambe gauche; une âme est emportée par un ange agenouillé sur la gauche de saint Michel; un second ange se devine avec la tête d'un diable en arrière plan. Sur le voûtain sud, subsiste une potence avec au moins quatre pendus visibles. Sur le quatrième voûtain, le soleil surmonte deux anges qui encadrent une scène centrale disparue. Ces scènes peuvent être datées de la fin du XV ou début du $\mathrm{XVI}^{\mathrm{e}}$ siècle par comparaison avec l'ange mis au jour dans la dernière travée du bas-côté sud. texte du Pèlerinage de l'âme de Guillaume de Digulleville, écrit vers le milieu du XIVe siècle, mais largement diffusé au siècle suivant. Cependant il est bien difficile de préciser comment s'est opéré le passage de l'écrit à l'image. Ces décors peints successifs témoignent d'une intense activité et de relations extérieures autour de cette église, qui apparaît dans les textes en 1131, dont deux seigneurs se partageaient au XII ${ }^{\mathrm{e}}$ et encore au $\mathrm{XVI}^{\mathrm{e}}$ siècle les honneurs du lieu.

Des peintures murales ont également été découvertes à l'église de Teigny, située à quelques kilomètres de la précédente. Le maire de la commune a fait réaliser des sondages sur les murs intérieurs de l'église Saint-Nazaire-et-Saint-Celse par Jean-Rémy Brigand, lors de son passage à Asnois. Plusieurs panneaux, chacun consacré à un ou deux saints ou saintes du XVI ${ }^{\mathrm{e}}$ siècle, furent découverts. Cette petite commune, en charge de la 
restauration de l'église non protégée, à l'instar de celle d'Asnois, a pu profiter à plusieurs reprises de nos conseils et de notre soutien ${ }^{17}$, dans un premier lieu pour l'assurer de la valeur des découvertes faites, puis sur la nécessité d'assurer l'entretien du bâtiment, notamment sa consolidation avant d'entreprendre la restauration finale des peintures murales.

Depuis 2000, l'un de nos fidèles partenaires est le Centre d'Études Médiévales d'Auxerre, CEM <lien vers http://www.cem-auxerre.fr>. Il a été demandé à l'association PACoB d'intervenir dans le cadre de leurs stages de relevés de peintures murales et du bâti organisés durant plusieurs années dans l'église Saint-Martin de Branches dans l'Yonne. Ce double stage, en lien avec les interventions de Laurence Blondaux, conservatricerestauratrice, a permis de mieux comprendre les phases de construction et reconstruction du bâtiment, d'établir une chronologie des enduits et les liens entre l'édifice lui-même et les différents décors de l'ensemble des murs. Car à la Procession de laïss, du bas-côté sud, connue depuis 1939 et classée M.H. le 4 juin 1973, se sont ajoutés des fragments découverts en 1990 lors de sondages effectués par I. Takahashi, restaurateur, dans l'ensemble de l'édifice.

Dans un premier temps, il est apparu que la Procession avançait vers un homme à cheval et vêtu de son armure (fig. $\mathbf{n}^{\circ} \mathbf{6}$ ) jusque-là caché par le badigeon moderne et que cette couche picturale était en lien direct avec la construction du bas-côté, postérieure à la première phase de construction de la nef. Dans cette dernière, sur le mur nord, un apôtre dans un médaillon tenant un livre et une croix de consécration était visible depuis des années. Les sondages de 1990 révélèrent une seconde couche picturale, plus ancienne et représentant un système de joints peints rouges sur fond blanc parsemé de fleurs à cinq lobes, aujourd'hui noires ${ }^{18}$ et des traces dans la partie supérieure du mur. Il était difficile jusque-là d'établir un lien entre les décors de la nef et la Procession du bas-côté sud. 


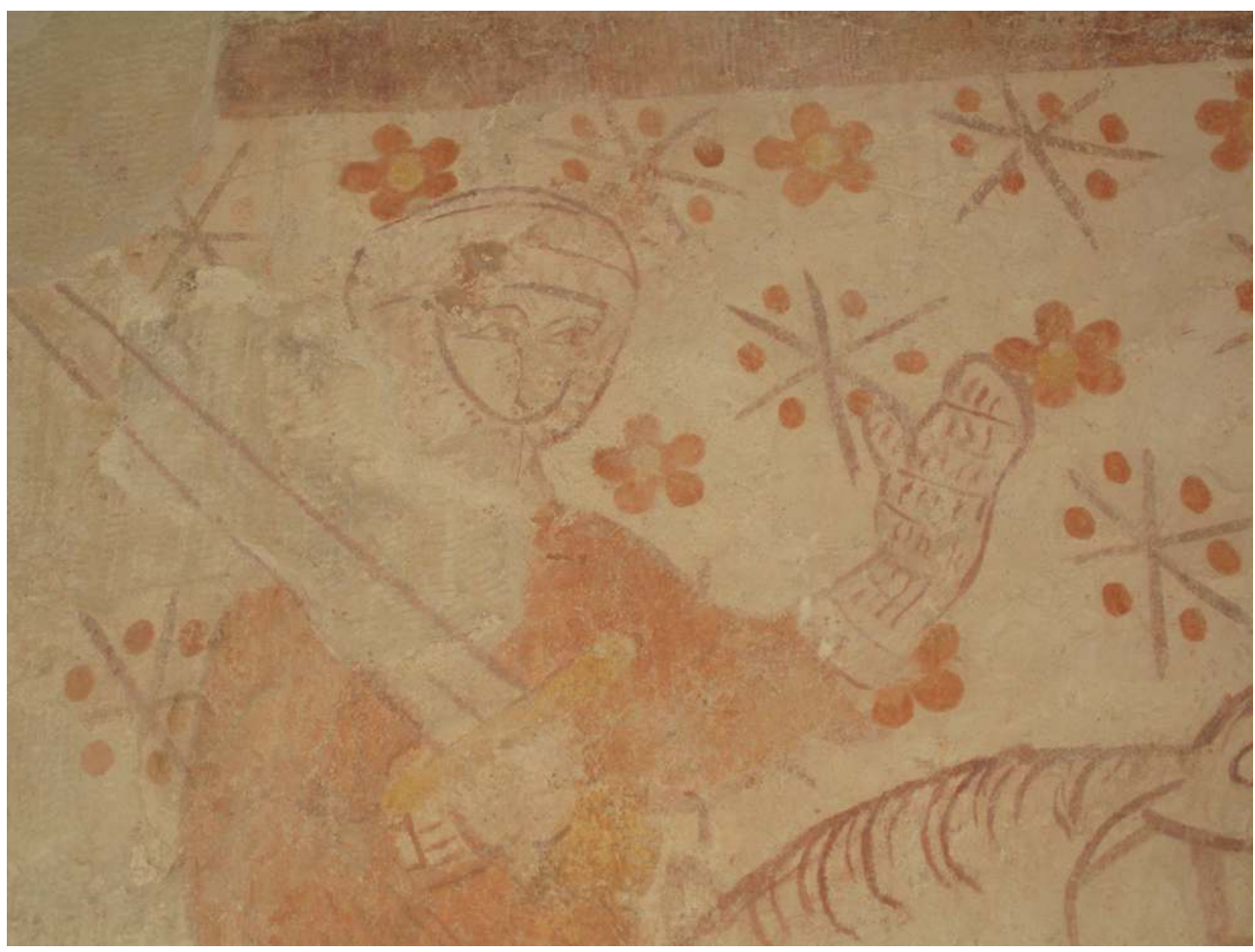

Branches (89), cavalier en armure saluant la procession d'un geste de la main gauche, bas-côté sud. Phot. Caffin, Marie-Gabrielle. (c) Caffin, Marie-Gabrielle.

Lors du dégagement des enduits modernes par Laurence Blondaux, un décor supplémentaire, moderne, est apparu, rendant encore plus difficile la compréhension. Le travail des stagiaires, sur plusieurs parties de l'église, a été tout d'abord de dissocier avec l'aide d'un professionnel, Carlos Castillo, les différentes couches picturales à l'aide des relevés puis de les analyser. La première couche picturale de la nef a été partiellement dégagée en parallèle par la conservatrice-restauratrice, permettant d'identifier un apôtre en médaillon, saint Barthélemy ${ }^{19}$, reconnaissable au couteau qu'il tient dans la main droite. Il a dans l'autre main la croix de consécration. Il est accompagné d'autres médaillons aujourd'hui peu lisibles et s'insère dans le réseau d'appareils peints en rouge garnis de fleurs, apparu dès 1990 et dégagé sur une grande surface. Les médaillons se situent à hauteur d'homme et le décor ornemental se poursuit sur toute la hauteur du mur, s'interrompant pour laisser la place à une frise de cavaliers bataillant devant une ville. Cette première couche appartient à la première phase d'ornementation de l'édifice au XIII ${ }^{e}$ siècle. Sur la seconde couche, l'apôtre en médaillon, visible depuis toujours, est accompagné de plusieurs frises historiées pouvant avoir été réalisées lors du remaniement de l'édifice et la construction du bas-côté sud en lien avec d'autres décors répartis dans l'ensemble de la nef et du chœur, expliquant la reprise du thème de l'apôtre tenant la croix de consécration.

Lors de ces stages, il a été demandé à l'association PACoB d'assurer une formation sur l'histoire et l'étude des peintures murales, sous la forme d'une journée en salle et de visites de différents sites à peintures murales, le CEM assurant la partie pratique, à savoir les relevés eux-mêmes. L'entreprise Blondel a été ensuite chargée de poursuivre le dégagement et la restauration des peintures murales de la nef. Ce chantier a été d'une 
très grande complexité puisque plusieurs couches picturales se juxtaposaient et des choix de conservation ont dû être faits par la conservatrice des Monuments historiques de Bourgogne, Cécile Ullmann. Une étude en cours actuellement sur l'édifice et l'ensemble des peintures murales devrait paraitre prochainement. C'est également le CEM qui nous a mis en relation avec la conservatrice-restauratrice Sylvie Mazillier qui, intervenant sur le chantier de l'église Saint-Sébastien de Bellefond (Côte-d'Or), s'interrogeait sur une iconographie particulière. Nous avons pu nous rencontrer, en 2008, à l'occasion de travaux de réfection entrepris dans l'édifice. Des sondages lui avaient été commandés par Dominique Jouffroy, architecte du patrimoine en charge du chantier. En octobre 2008, la conservatrice-restauratrice rend son rapport sur cette première campagne de sondages, constatant la présence très parcimonieuse de polychromie dans le chœur.

La seconde campagne révélera plusieurs scènes figurées, dont un maître autel peint en trompe-l'œil et des croix de consécration de deux tailles différentes positionnées en différents endroits : cinq sont de petite dimension, ornées aux extrémités d'une fleur de lys et huit de plus grand diamètre; deux sont peintes dans le chœur et six dans la nef. Dans le vaisseau central, un décor de boiseries peint en trompe-l'œil s'agence autour des deux autels latéraux. Sur le mur sud de la nef, de faux cartouches devaient appartenir à un ensemble ornemental plus complet englobant l'autel pour se poursuivre sur le mur sud jusqu'à la baie. Il servait donc à délimiter et mettre en valeur l'espace autour de l'autel. Au nord, deux figures de saints sont intégrées aux fausses boiseries. Saint Nicolas est représenté au-dessus de l'autel à gauche de l'arc triomphal tandis qu'un autre saint de plus grande dimension, non identifiable avec certitude, pourrait appartenir à une autre campagne d'exécution. Dans le chœur, un maître-autel est peint sur le mur du chevet plat englobant la baie centrale, bouchée probablement à l'occasion de la mise en place de cet ensemble. Sa composition à étages reprend le schéma des maîtres-autels disposés dans les chœurs des églises aux XVII et ${ }^{e} \mathrm{XVIII}^{\mathrm{e}}$ siècles. L'équilibre est parfaitement respecté avec un panneau central flanqué de deux statues peintes inscrites dans des niches, représentant saint Jean l'évangéliste et saint Maurice. Sur la partie supérieure, la figure du Christ occupe le centre, encadrée de chaque côté par un bouquet floral et une coupe de fruits. La scène principale dans la partie inférieure est plus énigmatique. Elle se déroule dans un paysage verdoyant avec sur le côté gauche un ange posé sur une nuée et tenant une palme dans la main (fig. $\mathbf{n}^{\circ} \mathbf{7}$ ). Il désigne ce qui pourrait être une couronne au milieu de rayons lumineux. Une statue ou un objet, aujourd'hui disparu, venait se positionner devant le soleil, sur une console dont la trace subsiste. Une inscription au-dessus de la scène est tirée du psaume 88 : Misericordias Domini in aeternum cantabo. Cette composition recouvre un appareil à joints peints, visible sur la gauche du chevet, appartenant à une campagne de réalisation antérieure. Le style des figures, des visages et le traitement des costumes, ainsi que la composition architecturale, témoignent d'une exécution au début du XVII siècle. Nous avons pu suivre de près ce chantier bénéficiant des remarques de la responsable et lui fournir des éléments d'identification et de datation pour son rapport. Ce travail sur le terrain avec les professionnels apparait primordial et devrait pouvoir se développer car nos regards croisés, sur les chantiers présentés ici de Bellefond et Branches, ont permis dans chacun des cas d'aller plus avant dans la connaissance et la compréhension de ce qui est mis en valeur. 


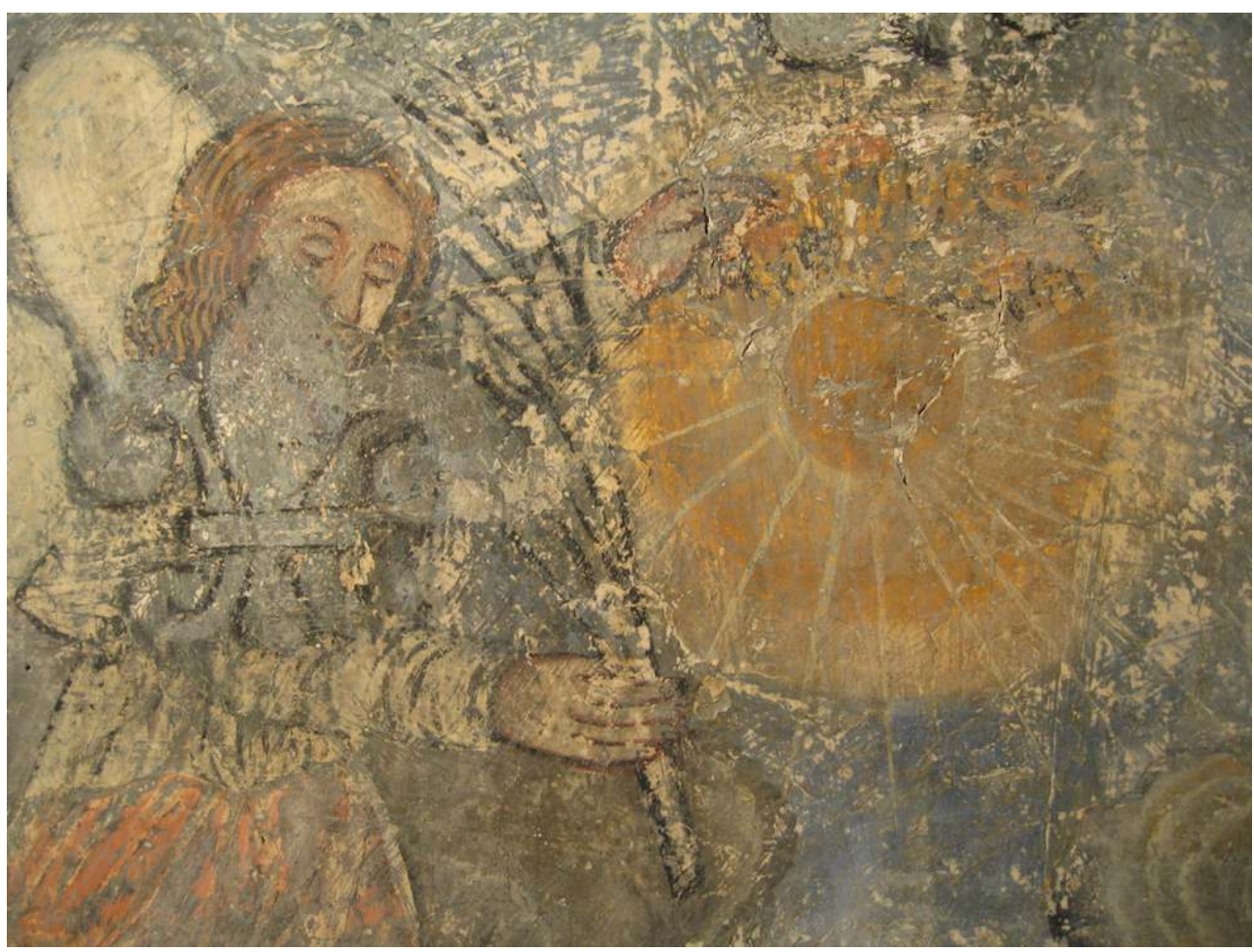

Bellefond (21), détail de l'ange présent sur la gauche dans le panneau central du maître-autel.

Phot. Caffin, Marie-Gabrielle. (c) Caffin, Marie-Gabrielle.

32 Au printemps 2009, les travaux de dégagement avaient débuté pour se poursuivre durant quelques mois. Les panneaux peints de la nef et les croix de consécration ont été entièrement restaurés. Une nouvelle campagne d'intervention pour la réintégration sera nécessaire dans l'avenir pour la mise en valeur du maittre-autel peint sur le mur du chœur. Une présentation des travaux par l'architecte du patrimoine et des peintures murales par un membre de l'association pendant les journées du patrimoine a permis de sensibiliser le public local à son patrimoine en lui en offrant une meilleure compréhension et de mobiliser les élus afin de poursuivre la restauration. En conclusion, il est important d'insister sur le fait que le recensement des peintures murales est un travail de longue haleine. Il n'est jamais achevé car l'actualité, nous venons de le percevoir, est toujours riche de nouvelles découvertes. Être à l'affût des nouveaux chantiers, des apparitions fortuites permet d'avoir un regard toujours neuf. Les tournées, les visites ponctuelles offrent la possibilité d'être attentif à l'état de conservation de ce patrimoine, à la diffusion de la connaissance et de l'information. Les partenariats, développés depuis plusieurs années, nous permettent aujourd'hui d'être de plus en plus présents au cœur de l'actualité régionale. Soulignons encore qu'il est très important de se mettre au service des acteurs en charge de la découverte et de la sauvegarde de ces décors peints pour pouvoir avancer dans la même direction, à savoir la compréhension et la mise en valeur de ce patrimoine fragile. 


\section{NOTES}

1. - Nous remercions Marianne Lussiez, étudiante à l'Université de Bourgogne, pour son aide dans la traduction.

2. - NEURY, Aimée. Notes sur les peintures murales de la France. Paris : Médiathèque du patrimoine, CRMH, enquête dactylographiée, 6 vol., 1942-1977. Se référer à l'article du GRPM dans cette même revue afin d'avoir une vision globale des enquêtes diverses menées régionalement.

3. - DESCHAMPS, Paul et THIBOUT, Marc. La peinture murale en France, le haut Moyen Âge et l'époque romane. Paris : Éd. d'histoire de l'art (Ars et Historia), 1951; BONNEFOY, Yves. Peintures murales de la France gothique. Paris : Hartmann, 1954 ; DESCHAMPS, Paul et THIBOUT, Marc. La peinture murale en France au début de l'époque gothique de Philippe Auguste à la fin du règne de Charles V (1180-1380). Paris : CNRS, 1963.

4. - D'ocre et d'azur : Peintures murales en Bourgogne. Dijon/Paris : Musée archéologique de Dijon/R. M. N., 1992.

5. - Mt 27, 46 : Mon Dieu, Mon Dieu, pourquoi m'as-tu abandonné ?

6. - Couleur de temps, fragments d'histoires. Dijon : PACoB, diffusion Éditions de l'Armançon, 2003.

7. - RUSSO, Daniel (dir.). Peintures murales médiévales, XIIe-XVIe siècle. Regards comparés. Dijon : EUD, Art \& Patrimoine, 2005.

8. - Les cahiers $P A C o B, n^{\circ} 1$ décembre $2006, n^{\circ} 2$ décembre 2007 et $n^{\circ} 3$ décembre 2010. Dijon : PACoB, a.pacob@wanadoo.fr.

9. - Cette identification a été discutée dans deux articles. La scène aurait mérité une étude, lors de sa dernière restauration par l'atelier ARCAMS, avec un examen sous ultra-violets pour confirmer ou non l'authenticité de certaines parties de la composition. CAFFIN, Marie-Gabrielle. "Inventaire des peintures murales en Côte-d'Or, État de la question au terme de la première enquête ». Mémoires de la Commission des Antiquités de la Côte-d'Or, 1987-1988, t. XXXV, p. 375-384 ; LAGABRIELLE, Sophie. "Nuits-Saint-Georges". D'ocre et d'azur: Peintures murales en Bourgogne. Dijon/Paris : Musée archéologique de Dijon/R. M. N., 1992, p. 148-149.

10. - Nous tenons à rendre hommage à Marie-Antoinette Stroeker, aujourd'hui disparue, qui a fait un travail sur le terrain essentiel pour la connaissance et la sauvegarde du mobilier liturgique. Laurence Joignerez, en charge du Musée d'Histoire et d'Archéologie de Nuits-SaintGeorges, a été une interlocutrice de premier plan pour l'étude des peintures murales de l'église Saint-Symphorien et nous pouvons souligner sa mobilisation constante pour la mise en valeur de ce patrimoine.

11. - D'ocre et d'azur, op. cit., 1992, D'ocre et d'azur: Peintures murales en Bourgogne. Dijon/Paris : Musée archéologique de Dijon/R. M. N., 1992, p. 280-281.

12. - « Rencontre avec Cécile Ulmann ». Les Cahiers PACoB, n³. Dijon : PACoB, 2007, p. 38-41.

13. - Couleur de temps, fragments d'histoires. Dijon : PACoB, diffusion Éditions de l'Armançon, 2003, p. 72-73.

14. - De terre \& d'esprit, Le patrimoine spirituel de la Côte-d'Or. Paris : Somogy éditions d'art et Dijon : Conseil Général de la Côte-d'Or, 2007.

15. - La Fédération REMPART Bourgogne/Franche-Comté a cette particularité d'ouvrir des chantiers bénévoles l'été visant à la restauration des peintures murales. Les personnes accueillies sont essentiellement des jeunes en quête d'un stage ou d'une formation dans le cadre de leur cursus universitaire lié aux métiers de la restauration. Si la nature de la découverte le permet, cela peut être d'une très grande aide financière et une source d'animation pour les petites communes. 
16. - L'association $\mathrm{PACOB}$ a participé à la rédaction de panneaux didactiques destinés à la présentation de l'édifice, des travaux et des décors à la fin de certains chantiers bénévoles et dernièrement a apporté son aide à la réalisation d'une exposition itinérante pour mettre en valeur leur travail en Bourgogne entrepris depuis plus de vingt ans.

17. - La présence d'un groupe de visiteurs s'intéressant à l'église et son décor peint a été une façon concrète pour le maire de légitimer l'importance des peintures vis-à-vis des élus et habitants de la commune.

18. - Ces fleurs devaient être à l'origine rouges. Ce rouge a viré lors du dégagement du badigeon et elles ont malheureusement été restaurées noires sans études sur la nature du pigment d'origine.

19. - CAFFIN, Marie-Gabrielle. «Les apôtres porteurs des croix de consécration ». Les cahiers PACoB, n², décembre 2007. Dijon : PACoB, p. 16-21.

\section{RÉSUMÉS}

De 1988 à 1992, une première opération de recensement des peintures murales, menée par le Service régional de l'Inventaire général de Bourgogne, a eu lieu à l'initiative du Conseil régional. Une exposition intitulée "D'ocre et d'Azur » et son catalogue permirent à un large public de découvrir la richesse de ce patrimoine. Dix ans après, cette enquête thématique régionale a été reprise pour être complétée grâce à plusieurs partenaires (le Service régional de l'Inventaire, le CNRS, l'Université de Bourgogne, le Conseil régional et l'association PACoB) et présentée à nouveau au public en 2003 au Musée archéologique de Dijon sous la forme d'une exposition temporaire et d'un catalogue, «Couleur de temps, fragments d'histoires », puis d'une exposition itinérante grâce à l'association $\mathrm{PAC} O \mathrm{~B}$. Un troisième volet d'enquête s'est ouvert grâce à une équipe soucieuse de la valorisation de ce patrimoine mural. En effet, de nouvelles découvertes continuent à enrichir notre connaissance des images peintes sur les murs des églises et des propriétés privées. À travers quelques exemples particulièrement marquants (Chassignelles (89), Branches (89), Villeneuve-les-Genêts (89), Diennes-Aubigny (58), Asnois (58), Bellefond (21), Nuits-Saint-Georges (21)), le lecteur pourra découvrir dans cet article la politique de conservation-restauration toujours féconde en Bourgogne et de nouveaux sites majeurs pour la compréhension de la création picturale du Moyen Âge au XVII ${ }^{\mathrm{e}}$ siècle.

From 1988 to 1992, a first census of wall-paintings led by the Service régional de l'Inventaire géneral of Burgundy took place, initiated by the Conseil Régional. An exhibition named "D'ocre et d'Azur" and its catalogue allowed the riches of this heritage to be discovered by a large public. Ten years later, this thematic survey of the region started again in order to be completed, with the help of several partners (Service Régional de l'Inventaire, the CNRS,University of Burgundy, Conseil Régional, association PACoB), and presented once again to the public in 2003, at the Musée Archéologique of Dijon, through a temporary exhibition accompanied by a catalogue, "Couleurs de temps, fragments d'histoire", then of a traveling exhibition thanks to the association PACOB. A team gave another development to this survey concerned with the value of this wall-painting heritage. Indeed, new discoveries keep widening and deepening the knowledge about these paintings in churches and private places. Through some particularly outstanding examples (Chassignelles (89), Branches (89), Villeneuve-les-Genêts (89), Diennes-Aubigny (58), Asnois (58), Bellefond (21), Nuits-Saint-Georges (21)), readers will be able to discover in this 
article the actual conservation and restoration policies in Burgundy as well as several major places regarding the understanding of pictural creation from the Middle-Ages to the $17^{\text {th }}$ century.

\section{AUTEURS}

\section{MARIE-GABRIELLE CAFFIN}

historienne de l'art

\section{FRANÇOISE PERROT}

CNRS

ALAIN RAUWEL

historien, Université de Bourgogne, membres de l'association PACoB caffinjpmg@wanadoo.fr 